\title{
ANÁLISE QUANTITATIVA DO PALATO DURO EM DIFERENTES TIPOLOGIAS FACIAIS DE RESPIRADORES NASAIS E ORAIS
}

\section{Quantitative analysis of the hard palate in different facial typologies in nasal and mouth breathers}

\author{
Luana Cristina Berwig (1), Ana Maria Toniolo da Silva ${ }^{(2)}$, Eliane Castilhos Rodrigues Côrrea (3), \\ Anaelena Bragança de Moraes ${ }^{(4)}$, Márlon Munhoz Montenegro ${ }^{(5)}$, Rodrigo Agne Ritzel ${ }^{(6)}$
}

\section{RESUMO}

Objetivo: comparar as dimensões do palato duro em diferentes tipologias faciais de crianças respiradoras nasais e orais. Método: a amostra foi constituída por 54 crianças, na faixa etária entre sete e 11 anos, distribuídas em grupos conforme o tipo facial e o modo respiratório. O tipo facial foi obtido por meio da análise cefalométrica de Ricketts, e o modo respiratório foi determinado a partir da avaliação fonoaudiológica e do diagnóstico otorrinolaringológico. Para realização de medidas transversais, verticais e do comprimento anteroposterior do palato duro, foram obtidos modelos em gesso do arco dental maxilar. Para comparação das dimensões do palato duro entre os grupos, foram utilizados testes paramétricos e não paramétricos, ao nível de significância de $5 \%$. Resultados: não foi verificada diferença estatisticamente significante nas medidas do palato duro entre as crianças braquifaciais, mesofaciais e dolicofaciais. Verificou-se diferença estatisticamente significante na distância entre os segundos pré-molares nos diferentes tipos faciais das crianças respiradoras nasais e orais, sendo que esta diferença não foi detectada nas comparações múltiplas. Conclusão: as dimensões do palato duro não diferiram quando analisadas em diferentes tipos faciais independente do modo respiratório. Porém, evidenciou-se diferença na distância entre os segundos pré-molares quando o tipo facial foi analisado nos respiradores nasais e orais.

DESCRITORES: Palato Duro; Medidas; Circunferência Craniana; Face; Respiração Bucal; Estudo Comparativo

(1) Fonoaudióloga Residente do Programa de Residência Multiprofissional Integrada em Gestão e Atenção Hospitalar no Sistema Público de Saúde da Universidade Federal de Santa Maria, UFSM, Santa Maria, RS, Brasil; Mestre em Distúrbios da Comunicação Humana pela Universidade Federal de Santa Maria.

(2) Fonoaudióloga; Professora Associada do Departamento de Fonoaudiologia da Universidade Federal de Santa Maria, UFSM, Santa Maria, RS, Brasil; Docente do Programa de Pós-Graduação em Distúrbios da Comunicação Humana da Universidade Federal de Santa Maria, UFSM, Santa Maria, RS, Brasil; Doutora em Ciências dos Distúrbios da Comunicação Humana pela Universidade Federal de São Paulo.

(3) Fisioterapeuta; Professora Adjunta do Departamento de Fisioterapia da Universidade Federal de Santa Maria, UFSM, Santa Maria, RS, Brasil; Docente do Programa de Pós-Graduação em Distúrbios da Comunicação Humana da Universidade Federal de Santa Maria, UFSM, Santa Maria, RS, Brasil; Doutora em Biologia Patologia Buco Dental pela Universidade Estadual de Campinas.

(4) Química industrial; Professora Adjunta do Departamento de Estatística da Universidade Federal de Santa Maria, UFSM, Santa Maria, RS, Brasil; Docente do Programa de Pós-Graduação em Distúrbios da Comunicação Humana da Universidade Federal de Santa Maria, UFSM, Santa Maria, RS, Brasil; Doutora em Epidemiologia pela Universidade Federal do Rio Grande do Sul.

(5) Cirurgião-dentista; Especialista em Periodontia; Mestrando do Programa de Pós-Graduação em Clínica Odontológica da Universidade Federal do Rio Grande do Sul, UFRGS, Porto Alegre, RS, Brasil.

(6) Médico otorrinolaringologista; Mestre em Distúrbios da Comunicação Humana pela Universidade Federal de Santa Maria, UFSM, Santa Maria, RS, Brasil.

Conflito de interesses: inexistente 


\section{INTRODUÇÃO}

As variações das estruturas que compõem o esqueleto craniofacial constituem a tipologia facial ${ }^{1}$. Uma das formas mais utilizadas para classificar o tipo facial mantém relação direta com o crescimento craniofacial e divide as faces em: braquifacial, na qual há tendência ao crescimento horizontal; mesofacial, que se caracteriza pelo crescimento equilibrado dos terços da face, não sendo necessárias adaptações funcionais; e dolicofacial, na qual há tendência ao crescimento vertical ${ }^{2}$.

Cada tipo facial apresenta características estéticas, ósseas, musculares e funcionais próprias ${ }^{1}$, sendo o palato duro uma das estruturas que pode apresentar seu formato diferenciado. A literatura refere que no tipo dolicofacial o palato duro é estreito e profundo, enquanto que no tipo braquifacial o palato duro é largo e raso ${ }^{2,3}$.

No tipo braquifacial, a coluna aérea também é mais larga e o terço inferior da face é menor, o que favorece a postura de repouso adequada dos lábios e da língua, possibilitando a respiração nasal. Em contraste, no tipo dolicofacial, a via aérea é mais estreita e o aumento do terço inferior da face dificulta o vedamento labial e o repouso da língua no palato duro, favorecendo a instalação da respiração oral $^{2}$.

O padrão de crescimento craniofacial predominantemente vertical é frequentemente associado à respiração oral, podendo ser causa ou consequência desta ${ }^{4,5}$. Caso a respiração oral se instale, alterações no sistema sensório-motor-oral podem ser verificadas, tais como as da morfologia do palato duro, que na literatura é caracterizado como: profundo e atrésico ${ }^{6,7}$; profundo e estreito8; ogival e estreito ${ }^{9}$; ogival ou em ogiva ${ }^{10-12}$ e profundo ${ }^{13}$.

Com base no exposto, observa-se que a morfologia do palato duro pode se modificar de acordo com o tipo facial e com o modo respiratório. No entanto, apesar de muitos estudos relatarem alterações morfológicas do palato duro, poucos têm analisado essa estrutura por meio de medidas quantitativas.

As medidas quantitativas do palato duro fornecem informações precisas sobre as características dessa estrutura. Ainda, os dados quantitativos podem ser comparados com as características clínicas, as quais são obtidas com base na formação e na experiência que o profissional tem para avaliar o palato duro, muitas vezes equivocada e controversa. Para minimizar tais equívocos, busca-se atualmente a inserção de métodos quantitativos que complementem a avaliação clínica orofacial.

Realizou-se esta pesquisa com 0 intuito de fornecer subsídios precisos quanto às características do palato duro em diferentes padrões faciais. Assim, o objetivo deste estudo foi comparar as dimensões do palato duro em diferentes tipologias faciais de crianças respiradoras nasais e orais.

\section{MÉTODO}

Este estudo apresenta caráter quantitativo transversal. Para realização do mesmo, foram triadas as crianças provenientes de quatro escolas públicas de Santa Maria - Rio Grande do Sul. As crianças assentiram a sua participação no estudo e tiveram o Termo de Consentimento Livre e Esclarecido assinado pelo responsável.

A triagem fonoaudiológica foi composta por anamnese com os pais e por inspeção orofacial. A anamnese verificou questões referentes aos dados de identificação, desenvolvimento geral, saúde geral, hábitos alimentares, hábitos orais, sono e tratamentos prévios e atuais. Por meio da inspeção orofacial, verificou-se a postura de repouso dos lábios, língua e mandíbula e o modo respiratório habitual.

Participaram do estudo as crianças que contemplavam os seguintes critérios de inclusão:

- gênero masculino ou feminino;

- faixa etária entre sete e 11 anos;

- fase de dentição mista;

- leucodermas;

- comparecer para realização da avaliação cefalométrica.

Os critérios de exclusão foram:

- históricos de tratamento fonoaudiológico e/ou ortodôntico e/ou ortopédico;

- sinais evidentes de comprometimento neurológico e/ou síndromes;

- limitações cognitivas;

- malformações craniofaciais.

Por meio desses critérios, foram selecionadas 54 crianças, $25(46,30 \%)$ meninos e 29 (53,70\%) meninas, com idade média de 9,44 $\pm 1,19$ anos.

As crianças selecionadas foram submetidas à avaliação cefalométrica, que possibilitou a classificação do tipo facial por meio do cálculo do índice VERT da análise cefalométrica de Ricketts ${ }^{14}$. O índice VERT é calculado pela média aritmética dos cinco valores de classificação facial: ângulo do eixo facial, profundidade facial, ângulo do plano mandibular, altura facial inferior e arco mandibular. De acordo com o índice VERT, as crianças foram distribuídas nos grupos: braquifacial (B), quando apresentavam valor do índice VERT maior que 0,5; mesofacial $(\mathrm{M})$, quando apresentavam o valor do índice VERT entre -0,5 e +0,5; e dolicofacial (D), 
quando apresentavam o valor do índice VERT menor que $-0,5$.

As crianças também foram submetidas à avaliação otorrinolaringológica para confirmação do modo respiratório, previamente classificado na inspeção orofacial. Esta avaliação foi composta por anamnese com os pais e exame clínico otorrinolaringológico, que incluiu oroscopia, rinoscopia anterior e otoscopia, seguidos de nasofibrofaringoscopia.

A partir das informações obtidas na triagem fonoaudiológica e na avaliação otorrinolaringológica, as crianças foram classificadas como respiradoras nasais e respiradoras orais. Foram consideradas respiradoras nasais as crianças que, apresentaram na inspeção orofacial modo respiratório nasal e na avaliação otorrinolaringológica ausência de sinais e sintomas de respiração oral diurna e/ou noturna. Foram consideradas respiradoras orais as crianças que, na inspeção orofacial, apresentaram modo respiratório oronasal ou oral e na avaliação otorrinolaringológica presença de sinais e sintomas de respiração oral diurna e/ou noturna.

A fim de contemplar os objetivos deste estudo, formaram-se os seguintes grupos de acordo com o tipo facial e o modo respiratório: grupo braquifacial com respiração nasal (BRN); grupo mesofacial com respiração nasal (MRN); grupo dolicofacial com respiração nasal (DRN); grupo braquifacial com respiração oral (BRO); grupo mesofacial com respiração oral (MRO); grupo dolicofacial com respiração oral (DRO).

Para que fossem obtidas as medidas quantitativas do palato duro, foi realizada avaliação odontológica com as crianças. Nesta avaliação foi efetuada a moldagem do arco dental maxilar com alginato para obtenção dos modelos em gesso, que foram estudados por meio de medidas transversais (largura), verticais (profundidade) e da medida do comprimento anteroposterior do palato duro.

Para a realização das mensurações, foram marcados pontos de referências nos modelos de gesso. Ao nível dos dentes caninos, primeiros e segundos pré-molares, os pontos foram marcados na borda gengival mais apical ${ }^{15}$. Na região dos primeiros molares, os pontos foram marcados na união da borda gengival com o sulco palatino ${ }^{16}$. $\mathrm{Na}$ região mais anterior do palato duro, o ponto foi marcado na linha sagital entre os incisivos centrais superiores ${ }^{15}$.
As medidas foram realizadas com paquímetro digital da marca Western ${ }^{\circledast}$, com resolução de 0,01 $\mathrm{mm}$ e precisão de $\pm 0,02 \mathrm{~mm}$. Os bicos do instrumento para medição interna foram utilizados para obtenção das medidas transversais e do comprimento anteroposterior do palato duro. Para a realização das medidas verticais, um fio de aço inox de $0,05 \mathrm{~mm}$ foi cortado com alicate ortodôntico no comprimento correspondente à medida transversal obtida e fixado com cera utilidade entre os pontos ao nível de cada um dos dentes considerados. Depois de fixado o fio, a profundidade era mensurada com a vareta do paquímetro.

O paquímetro era zerado antes da realização de cada medida e, quando a mesma era obtida, o valor da escala digital, considerado neste estudo, era conferido com o valor correspondente na escala analógica. Caso fossem divergentes, a medida era repetida, desconsiderando-se o primeiro valor obtido.

As seguintes medidas do palato duro foram mensuradas, conforme a subsequente ordem de realização:

Distância entre os caninos: distância transversal em milímetros entre os pontos da região dos caninos superiores (Figura 1).

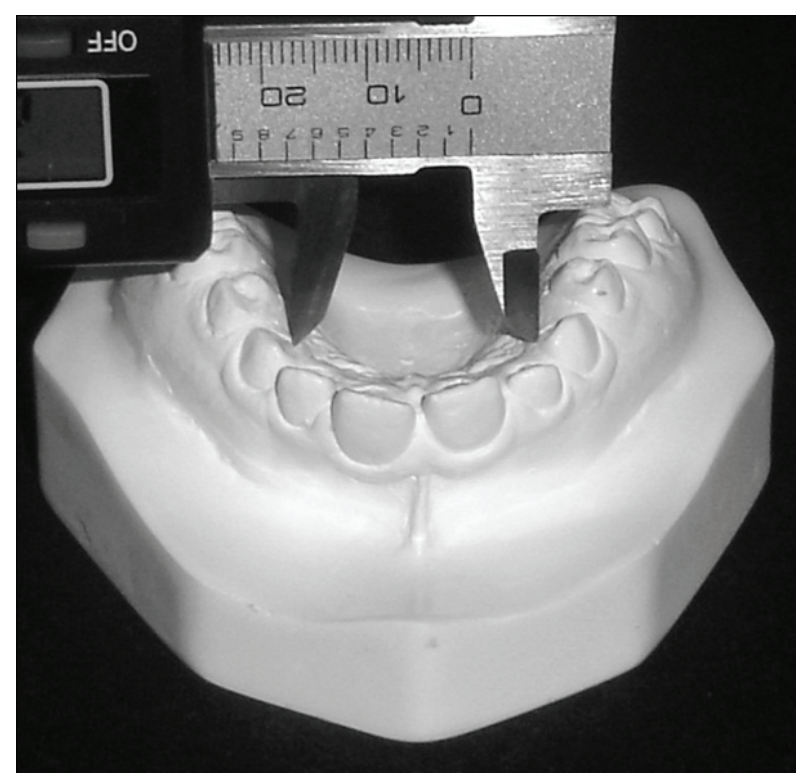

Figura 1 - Distância entre os caninos 
Profundidade ao nível dos caninos: medida vertical em milímetros da linha média palatina até o fio de aço inox que une a região dos caninos superiores (Figura 2).

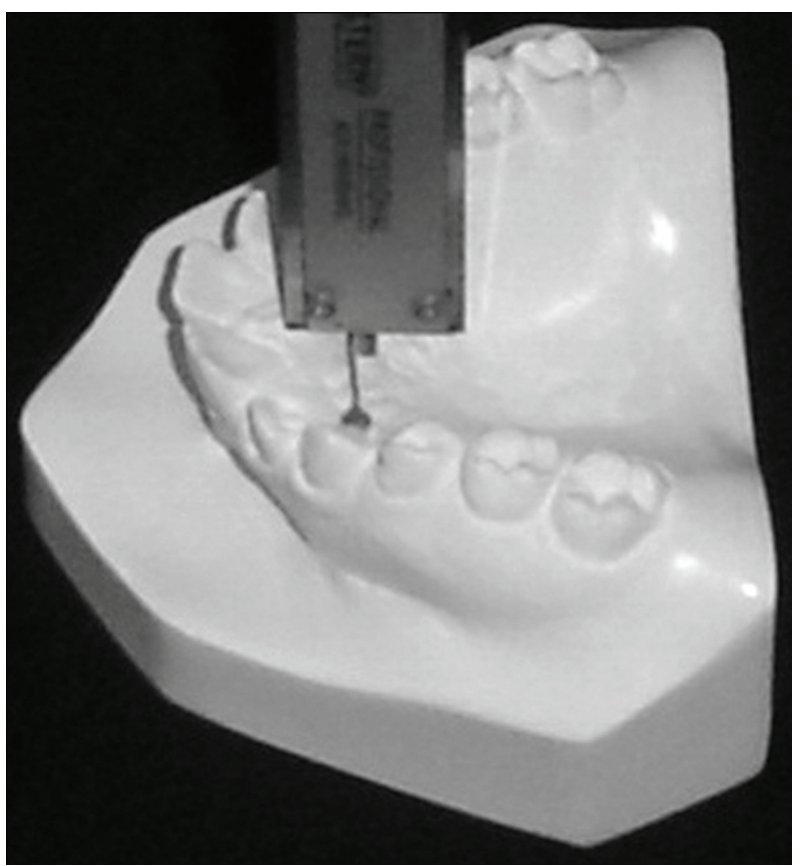

Figura 2 - Profundidade ao nível dos caninos

Distância entre os primeiros pré-molares: distância transversal em milímetros entre os pontos dos primeiros pré-molares superiores (Figura 3 ).

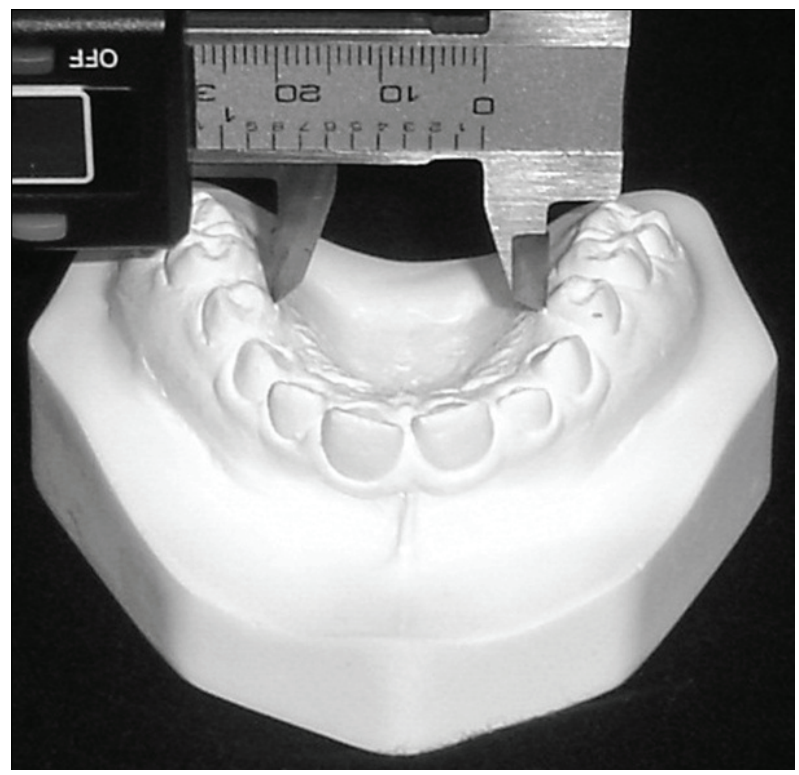

Figura 3-Distância entre os primeiros pré-molares
Profundidade ao nível dos primeiros pré-molares: medida vertical em milímetros da linha média palatina até o fio de aço inox que une a região dos primeiros pré-molares superiores (Figura 4).

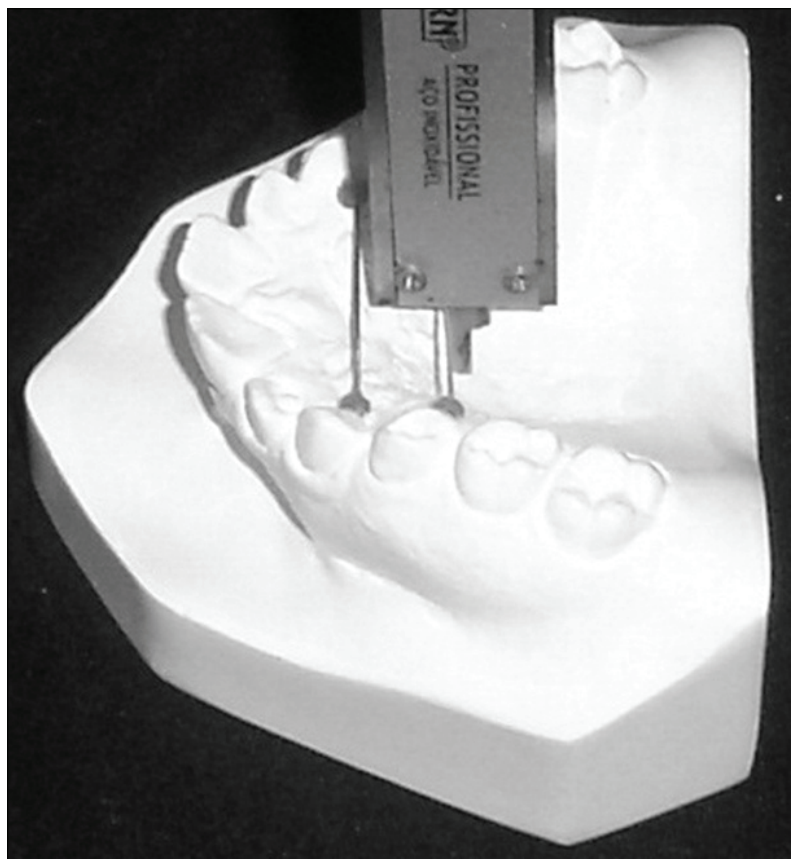

Figura 4 - Profundidade ao nível dos primeiros pré-molares

Distância entre os segundos pré-molares: distância transversal em milímetros dos pontos da região dos segundos pré-molares superiores (Figura 5).

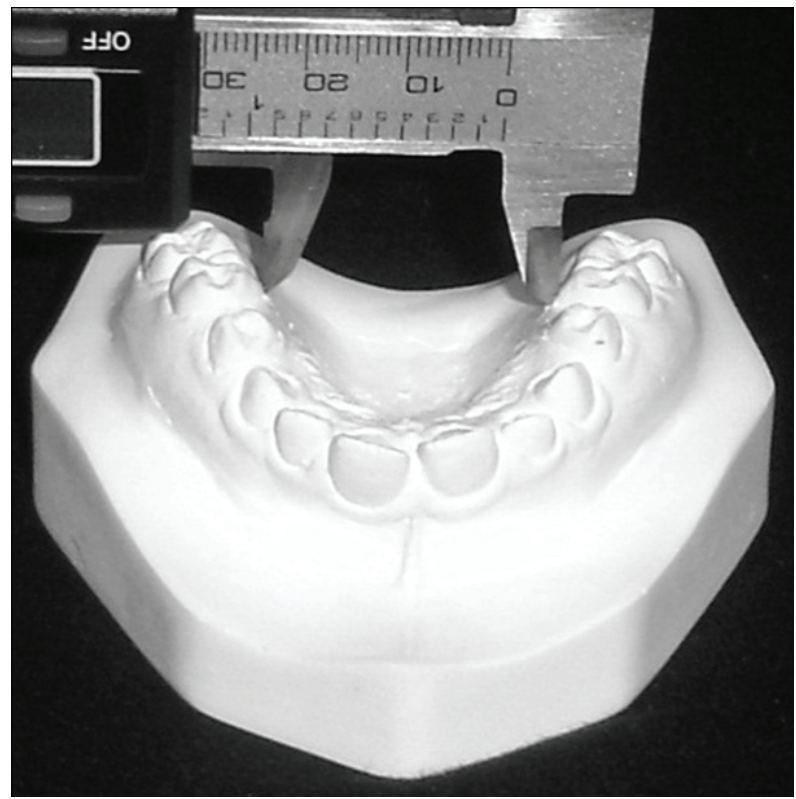

Figura 5-Distância entre os segundos pré-molares 
Profundidade ao nível dos segundos prémolares: medida vertical em milímetros da linha média palatina até o fio de aço inox que une a região dos segundos pré-molares superiores (Figura 6).

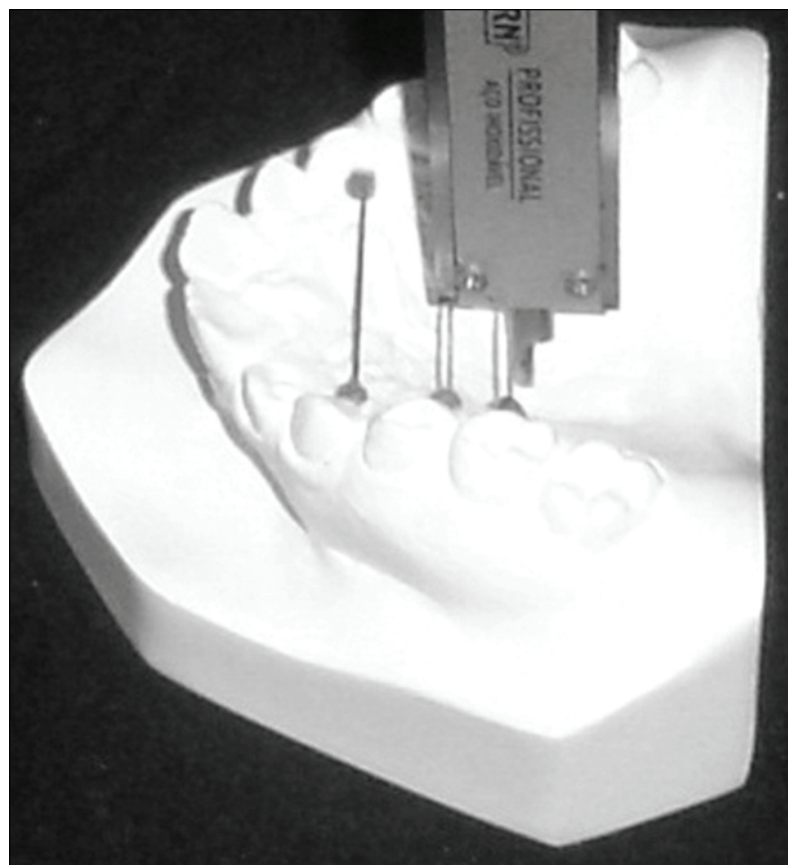

Figura 6 - Profundidade ao nível dos segundos pré-molares

Distância entre os primeiros molares: distância transversal em milímetros entre os pontos dos primeiros molares superiores (Figura 7).

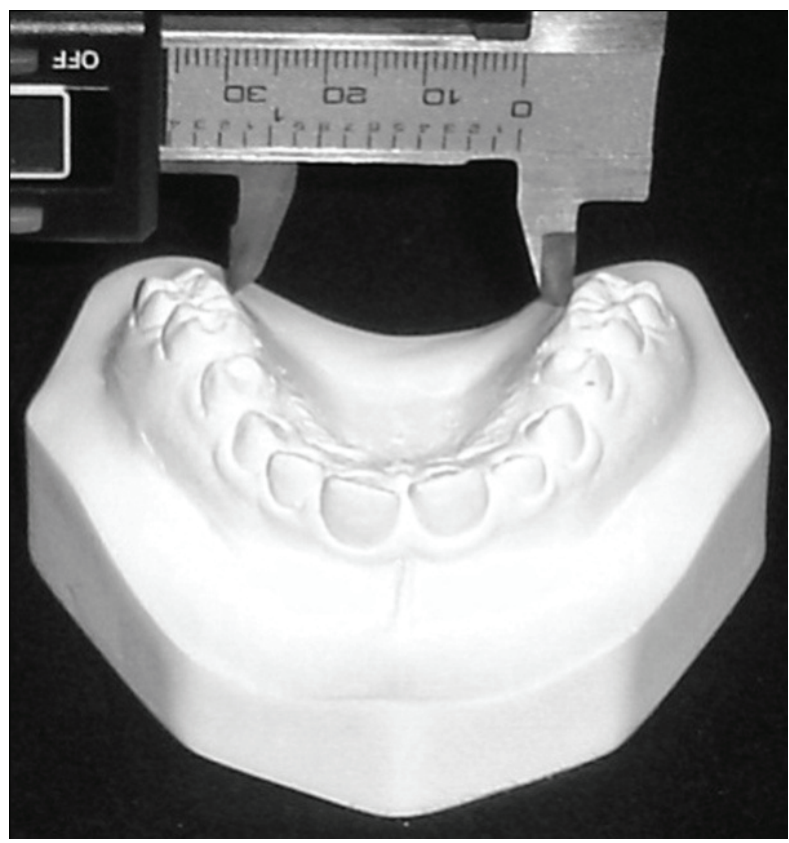

Figura 7 - Distância entre os primeiros molares
Profundidade ao nível dos primeiros molares: medida vertical em milímetros da linha média palatina até o fio de aço inox que une a região dos primeiros molares superiores (Figura 8).

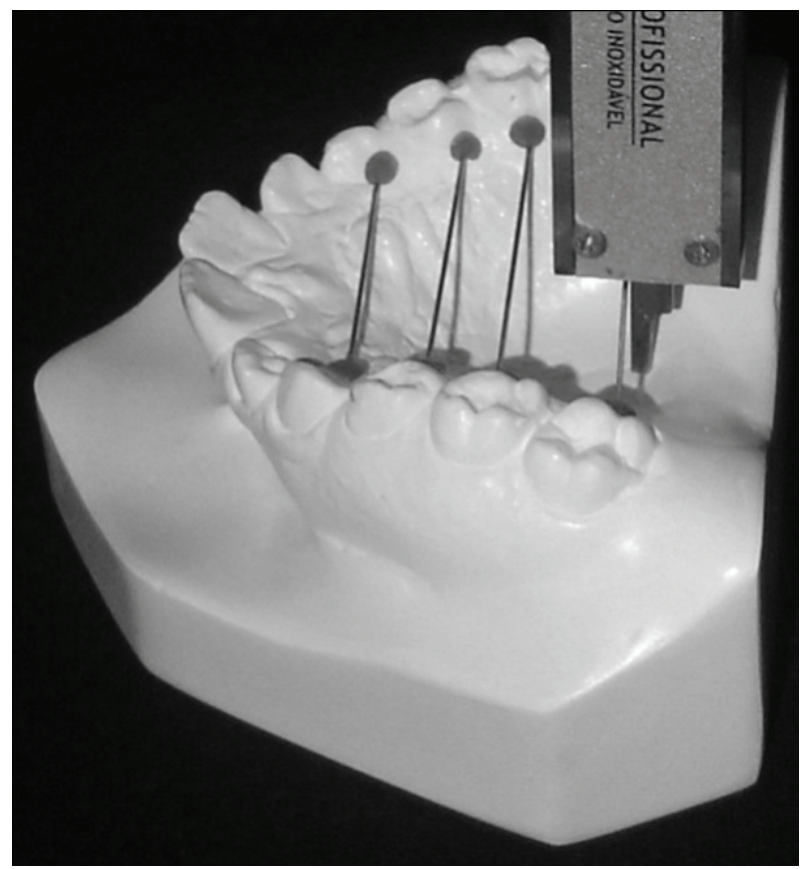

Figura 8 - Profundidade ao nível dos primeiros molares

Comprimento anteroposterior: medida da distância entre o ponto mais anterior do palato duro até o fio de aço inox que une a região dos primeiros molares superiores.

O valor $0,05 \mathrm{~mm}$, correspondente ao diâmetro do fio de aço inox, foi subtraído das quatro medidas verticais e do comprimento anteroposterior do palato duro.

Os pontos não foram marcados e as medidas não foram realizadas quando um ou ambos os dentes, considerados para qualquer uma das medidas supracitadas, não estivessem erupcionados.

Após 30 dias, as medidas do palato duro foram repetidas em $30 \%$ dos modelos $(n=23)$ selecionados aleatoriamente. A concordância entre a primeira e a segunda medida foi verificada por meio do cálculo do Coeficiente de correlação Intraclasse (ICC).

O presente estudo foi registrado e aprovado pelo Comitê de Ética em Pesquisa da instituição de origem sob o protocolo de número 220.0.243.000-8.

Por meio do teste de Lilliefors, verificou-se que as medidas da profundidade ao nível dos caninos e primeiros molares não apresentaram distribuição normal, sendo necessária a aplicação de testes paramétricos e não paramétricos. Na comparação das dimensões do palato duro entre os grupos B, M, 
D e entre os grupos BRN, MRN, DRN, BRO, MRO, DRO, foi utilizada análise de variância (ANOVA) e o teste de Kruskal-Wallis. Quando verificada diferença significante nesses últimos testes, foram realizadas comparações múltiplas por meio do teste de Tukey. Nas análises estatísticas, foi utilizado nível de significância de $5 \%(p<0,05)$.

As análises foram realizadas utilizando-se o software SPSS versão 17.0 (2008).

\section{RESULTADOS}

Verificou-se por meio do cálculo do Coeficiente de correlação Intraclasse (ICC) concordância significante entre as duas medidas para todas as mensurações do palato duro.

Os resultados da Tabela 1 não evidenciaram diferença nas dimensões do palato duro entre os diferentes tipos faciais.

Tabela 1 - Comparação das dimensões do palato duro entre os tipos faciais

\begin{tabular}{|c|c|c|c|c|}
\hline \multirow{3}{*}{ Dimensões do palato duro } & \multicolumn{3}{|c|}{ Grupos } & \multirow{3}{*}{$\mathbf{p}$} \\
\hline & $\begin{array}{l}\text { Braquifacial } \\
\quad(n=11)\end{array}$ & $\begin{array}{l}\text { Mesofacial } \\
(n=21)\end{array}$ & $\begin{array}{c}\text { Dolicofacial } \\
(n=22)\end{array}$ & \\
\hline & $\bar{X} \pm S$ & $\bar{X} \pm S$ & $\bar{X} \pm S$ & \\
\hline Distância entre os caninos & $26,71 \pm 1,90$ & $26,15 \pm 2,80$ & $26,81 \pm 2,60$ & 0,715 \\
\hline Profundidade ao nível dos caninos & $6,64 \pm 2,11$ & $7,47 \pm 2,28$ & $6,77 \pm 2,87$ & 0,626 \\
\hline Distância entre os $1^{\text {os }}$ pré-molares & $28,56 \pm 1,89$ & $28,16 \pm 2,49$ & $26,86 \pm 2,61$ & 0,105 \\
\hline Profundidade ao nível dos $1^{\text {os }}$ pré-molares & $11,62 \pm 1,16$ & $11,77 \pm 1,52$ & $12,35 \pm 1,82$ & 0,358 \\
\hline Distância entre os $2^{\text {os }}$ pré-molares & $32,18 \pm 2,22$ & $31,56 \pm 2,33$ & $30,40 \pm 2,36$ & 0,088 \\
\hline Profundidade ao nível dos $2^{o s}$ pré-molares & $12,25 \pm 1,19$ & $12,09 \pm 1,35$ & $12,93 \pm 1,70$ & 0,164 \\
\hline Distância entre os $1^{\text {os }}$ molares & $36,52 \pm 2,95$ & $35,75 \pm 2,43$ & $35,09 \pm 2,94$ & 0,367 \\
\hline Profundidade ao nível dos $1^{\text {os }}$ molares & $10,23 \pm 1,77$ & $10,37 \pm 1,72$ & $10,80 \pm 1,92$ & 0,738 \\
\hline Comprimento anteroposterior & $35,09 \pm 2,82$ & $34,48 \pm 1,88$ & $34,76 \pm 2,02$ & 0,745 \\
\hline
\end{tabular}

$\overline{\mathrm{X}}=$ média; $\mathrm{S}=$ desvio padrão; $\mathrm{p}<0,05$

$\mathrm{Na}$ Tabela 2, verificou-se diferença estatisticamente significante $(p<0,05)$ na distância entre os segundos pré-molares entre os grupos. Essa diferença não foi detectada nas comparações múltiplas realizadas por meio do teste de Tukey.

\section{DISCUSSÃO}

Esta pesquisa verificou as dimensões do palato duro nos planos transversal e vertical ao nível dos dentes caninos, primeiros e segundos pré-molares e primeiros molares, bem como o comprimento anteroposterior do palato duro. Como não foram encontradas pesquisas, relacionadas ao tipo facial e ao modo respiratório, que tenham realizado todas essas aferições, este estudo teve por base a metodologia de uma pesquisa que mensurou as dimensões do palato duro na síndrome de Turner ${ }^{15}$.

No levantamento bibliográfico realizado, verificou-se uma escassez de estudos que tenham examinado a morfologia do palato duro por meio de medidas quantitativas de acordo com a tipologia facial. Em vista disso, os resultados deste estudo foram contrastados com pesquisas relacionadas às dimensões do arco dental maxilar, que são aferidas com base nos pontos de referência em uma determinada região anatômica do dente ${ }^{3,17,18}$.

Por outro lado, foram encontradas pesquisas que aferiram as dimensões do palato duro em diferentes modos respiratórios, ou seja, que apresentaram os pontos de referência para realização das medidas na borda gengival. Nestas observou-se que outros instrumentos além do paquímetro foram empregados, como o Compasso tridimensional Korkhaus $^{19-21}$, que foi utilizado para realização de medidas em modelos de gesso e intraoralmente.

Foram incluídas neste estudo somente as crianças leucodermas, tendo em vista que variações nas estruturas craniofaciais podem ocorrem em diferentes raças ${ }^{22}$. É importante salientar ainda que as crianças foram classificadas como respiradoras nasais e orais, uma vez que vários estudos verificaram diferença estatisticamente significante nas dimensões do palato duro de crianças com diferentes modos respiratórios ${ }^{19-21}$. Em vista disso, optou-se por comparar as dimensões do palato duro em diferentes tipos faciais independente do modo respiratório (Tabela 1), bem como em diferentes tipos faciais de respiradores nasais e orais 
Tabela 2 - Comparação das dimensões do palato duro entre os tipos faciais de crianças respiradoras nasais e orais

\begin{tabular}{|c|c|c|c|c|c|c|c|}
\hline \multirow{3}{*}{$\begin{array}{l}\text { Dimensões do } \\
\text { palato duro }\end{array}$} & \multicolumn{6}{|c|}{ Grupos } & \multirow{3}{*}{ p } \\
\hline & $\begin{array}{l}\text { BRN } \\
(n=6)\end{array}$ & $\begin{array}{l}\text { MRN } \\
(n=8)\end{array}$ & $\begin{array}{l}\text { DRN } \\
(n=4)\end{array}$ & $\begin{array}{l}\text { BRO } \\
(n=5)\end{array}$ & $\begin{array}{l}\text { MRO } \\
(n=13)\end{array}$ & $\begin{array}{l}\text { DRO } \\
(n=18)\end{array}$ & \\
\hline & $\bar{X} \pm S$ & $\bar{X} \pm S$ & $\bar{X} \pm S$ & $\bar{x} \pm S$ & $\bar{X} \pm S$ & $\bar{X} \pm S$ & \\
\hline $\begin{array}{l}\text { Distância entre os } \\
\text { caninos }\end{array}$ & $27,29 \pm 1,67$ & $27,18 \pm 2,96$ & $26,17 \pm 2,65$ & $26,14 \pm 2,13$ & $25,49 \pm 2,62$ & $26,93 \pm 2,66$ & 0,665 \\
\hline $\begin{array}{l}\text { Profundidade ao } \\
\text { nível dos caninos }\end{array}$ & $6,94 \pm 1,89$ & $7,10 \pm 1,77$ & $6,33 \pm 3,81$ & $6,35 \pm 2,49$ & $7,70 \pm 2,60$ & $6,85 \pm 2,81$ & 0,914 \\
\hline $\begin{array}{l}\text { Distância entre os } \\
1^{\text {ss }} \text { pré-molares }\end{array}$ & $29,02 \pm 2,30$ & $29,19 \pm 2,87$ & $26,46 \pm 0,88$ & $27,99 \pm 1,25$ & $27,53 \pm 2,10$ & $26,95 \pm 2,87$ & 0,201 \\
\hline $\begin{array}{l}\text { Profundidade ao } \\
\text { nível dos } 1^{\text {os }} \text { pré- } \\
\text { molares }\end{array}$ & $11,80 \pm 1,55$ & $11,76 \pm 1,92$ & $11,06 \pm 2,11$ & $11,42 \pm 0,50$ & $11,78 \pm 1,31$ & $12,64 \pm 1,68$ & 0,372 \\
\hline $\begin{array}{l}\text { Distância entre } 2^{\circ S} \\
\text { pré-molares }\end{array}$ & $33,10 \pm 2,21$ & $32,85 \pm 2,18$ & $29,57 \pm 1,43$ & $31,07 \pm 1,83$ & $30,76 \pm 2,11$ & $30,58 \pm 2,51$ & $0,038^{*}$ \\
\hline $\begin{array}{l}\text { Profundidade ao } \\
\text { nível dos } 2^{\circ s} \text { pré- } \\
\text { molares }\end{array}$ & $12,55 \pm 1,34$ & $12,24 \pm 1,81$ & $11,43 \pm 1,32$ & $11,89 \pm 1,00$ & $12,01 \pm 1,05$ & $13,27 \pm 1,62$ & 0,095 \\
\hline $\begin{array}{l}\text { Distância entre } \\
\text { os } 1^{\circ s} \text { molares }\end{array}$ & $37,86 \pm 3,04$ & $36,74 \pm 2,18$ & $34,52 \pm 1,84$ & $34,92 \pm 2,09$ & $35,15 \pm 2,45$ & $35,22 \pm 3,16$ & 0,220 \\
\hline $\begin{array}{l}\text { Profundidade ao } \\
\text { nível dos } 1^{\text {os }} \\
\text { molares }\end{array}$ & $10,75 \pm 1,80$ & $10,42 \pm 2,19$ & $10,15 \pm 0,07$ & $9,59 \pm 1,69$ & $10,34 \pm 1,46$ & $10,95 \pm 2,10$ & 0,861 \\
\hline $\begin{array}{l}\text { Comprimento } \\
\text { anteroposterior }\end{array}$ & $33,75 \pm 2,60$ & $33,64 \pm 1,16$ & $34,40 \pm 2,09$ & $36,69 \pm 2,36$ & $34,99 \pm 2,09$ & $34,84 \pm 2,06$ & 0,155 \\
\hline
\end{tabular}

$\overline{\mathrm{X}}=$ média; $\mathrm{S}=$ desvio padrão; $p<0,05$; *significância pela Análise de Variância $(p<0,05)$; $B R N=$ grupo braquifacial com respiração nasal; $\mathrm{MRN}=$ grupo mesofacial com respiração nasal; $\mathrm{DRN}=$ grupo dolicofacial com respiração nasal; $\mathrm{BRO}=\mathrm{grupo}$ braquifacial com respiração oral; $\mathrm{MRO}=$ grupo mesofacial com respiração oral; $\mathrm{DRO}=$ grupo dolicofacial com respiração oral

(Tabela 2). Observou-se que os estudos que compararam as dimensões do arco dental maxilar entre diferentes tipos faciais, não levaram em consideração o modo respiratório apresentando pelo paciente ${ }^{3,17,18}$.

Com relação aos resultados, na comparação das dimensões do palato duro entre os diferentes tipos faciais, verificou-se que as médias das dimensões transversais foram menores e as verticais maiores nas crianças dolicofaciais ao nível dos primeiros pré-molares, segundos pré-molares e primeiro molares, enquanto que nas crianças braquifaciais houve aumento das médias transversais e a diminuição das verticais ao nível desses mesmos dentes. Apesar disso, não foi possível afirmar que a tipologia facial influencie nas dimensões do palato duro, uma vez que não foi encontrada diferença estatisticamente significante na comparação entre os grupos (Tabela 1).

Outros estudos também não encontraram relação entre a tipologia facial e as dimensões transversais do arco dental maxilar ao nível dos dentes que serviram de referência para obtenção das medidas do palato duro na presente pesquisa ${ }^{3,17,18}$. Já na comparação da medida vertical do arco dental maxilar ao nível dos primeiros molares entre diferentes tipos faciais, um destes estudos verificou diferença entre os grupos braquifacial e dolicofacial, sendo a medida vertical maior no segundo grupo ${ }^{3}$. A amostra dessa pesquisa foi constituída por pacientes na faixa etária entre 12 e 21 anos, o que pode justificar a divergência em relação aos achados do presente estudo.

$\mathrm{Na}$ comparação das dimensões do palato duro entre os diferentes tipos faciais de crianças respiradoras nasais e orais, foi verificada diferença estatisticamente significante na distância entre os segundos pré-molares (Tabela 2). Esta diferença não pôde ser detectada nas comparações múltiplas, provavelmente porque as crianças foram distribuídas em seis grupos, o que implicou em um número reduzido de crianças em alguns dos grupos formados.

Não foram encontrados outros estudos com metodologia semelhante que tenham comparado as dimensões do palato duro ou do arco dental maxilar em grupos constituídos a partir da tipologia facial e do modo respiratório, o que impossibilitou a comparação dos achados deste estudo com a literatura.

De modo geral, a média na distância entre os segundos pré-molares dos grupos formados a partir da tipologia facial de respiradores nasais e orais diminuiu do grupo BRN em direção ao grupo DRN e do grupo BRO para o DRO. 
Também foi possível verificar que as crianças do grupo BRN apresentaram a maior média na largura do palato duro ao nível dos segundos pré-molares, enquanto as crianças dos grupos DRN, seguido de $\mathrm{DRO}$, apresentaram as menores médias na largura ao nível destes dentes.

As modificações na morfologia do palato duro podem decorrer da conformação e tensão da musculatura orofacial, que podem variar de acordo com o tipo facial e o modo respiratório que o indivíduo apresenta. A musculatura estirada e menos potente, característica do tipo dolicofacial ${ }^{2}$, e a diminuição na tensão dos músculos orofaciais, frequentemente observada no respirador oral ${ }^{10}$, faz com que os tecidos moles exerçam menor força sobre os tecidos ósseos que compõe a face, podendo alterar o crescimento e desenvolvimento do esqueleto craniofacial ${ }^{23}$, incluindo a maxila e o palato duro.

Com base nos resultados encontrados e nos achados de outros estudos ${ }^{19-21,} 24$, acredita-se que a respiração oral tenha maior influência sobre as modificações na morfologia do palato duro em relação à tipologia facial, uma vez que a respiração nasal tem papel fundamental no desenvolvimento do terço médio da face ${ }^{25}$, região orofacial em que se encontram a maxila e o palato duro. Ainda assim, não se descarta a hipótese de que a magnitude das modificações na morfologia do palato duro também dependa da tipologia facial, ou seja, caso o indivíduo apresente respiração oral e padrão de crescimento predominantemente vertical, poderá apresentar maiores alterações nesta estrutura quando comparado com respirador oral braquifacial ou mesofacial. Entretanto, salienta-se que para a confirmação desta hipótese, faz-se necessária a realização de novas pesquisas com amostras maiores e metodologia semelhante, que forneçam novos subsídios sobre as dimensões do palato duro em diferentes tipologias faciais de respiradores nasais e orais.

\section{CONCLUSÃO}

A partir dos resultados desta pesquisa, foi possível concluir que:

- As dimensões do palato duro não diferiram quando analisadas em diferentes tipos faciais independente do modo respiratório;

- Evidenciou-se diferença na distância entre os segundos pré-molares quando o tipo facial foi analisado nos respiradores nasais e orais.

\section{AGRADECIMENTOS}

À Coordenação de Aperfeiçoamento de Pessoal de Nível Superior pela concessão de bolsa.

\section{ABSTRACT}

Purpose: to compare the hard palate dimensions in nasal and mouth breathing children of different facial typologies. Method: the sample comprised 54 children between seven and 11 year old, divided in two groups according to the facial type and breathing mode. The facial type was obtained through the Ricketts cephalometric analysis, and the breathing mode was determined from speech-language evaluation and otorhynolaryngologic diagnosis. In order to measure the transverse, vertical and anteroposterior length of the hard palate, plaster cast models of the children superior dental arch were obtained. To get the hard palate dimensions among the groups, parametric and nonparametric tests were used under a significance level of $5 \%$. Results: no statistically significant difference was found in hard palate measures among brachyfacial, mesofacial and dolichofacial children. A significant statistical difference in the distance among the second premolars was identified when compared with the different facial types of nasal and mouth breathing children, and such a difference was not detected in multiple comparisons. Conclusion: the dimensions of the hard palate did not show any difference when analyzed in different facial types regardless of the breathing mode. However, when analyzing the facial type in nasal and mouth breathers, we found a difference in the distance among the second premolars.

KEYWORDS: Palate, Hard; Measures; Cephalometry; Face; Mouth Breathing; Comparative Study 


\section{REFERÊNCIAS}

1. Ramires RR, Ferreira LP, Marchesan IQ, Cattoni DM, Andrada e Silva MA. Tipologia facial aplicada à Fonoaudiologia: revisão de literatura. Rev Soc Bras Fonoaudiol. 2010;15(1):140-5.

2. Bianchini EMG. Avaliação fonoaudiológica da motricidade oral - distúrbios miofuncionais orofaciais ou situações adaptativas. Rev Dental Press Ortodon Ortop Facial. 2001;6(3):73-82.

3. Esteves A, Bommarito S. Avaliação da profundidade do palato e das dimensões do arco dentário superior em indivíduos com má oclusão e diferentes tipos faciais. R Dental Press Ortodon Ortop Facial. 2007;12(4):84-98.

4. Castro AMAd, Vasconcelos MHF. Avaliação da infuência do tipo facial nos tamanhos dos espaços aéreos nasofaríngeo e bucofaríngeo. $R$ Dental Press Ortodon Ortop Facial. 2008;13(6):43-50.

5. Castro AMAd, Teles RP. Influência do tipo facial no tamanho do espaço aéreo nasofaríngeo. Orto SPO. 2008;41(4):393-8.

6. Barbosa MdC, Knop LAH, Lessa MM, Araujo TM. Avaliação da radiografia cefalométrica lateral como meio de diagnóstico da hipertrofia de adenóide. Rev Dent Press Ortodon Ortopedi Facial. 2009;14(4):83-91.

7. Berwig LC, Silva AMTd, Busanello AR, Almeida FLd, Bolzan GdP, Hennig TR, et al. Alterações no modo respiratório, na oclusão e na fala em escolares: ocorrências e relações. Rev CEFAC. 2010;12(5):795-802.

8. Bianchini AP, Guedes ZC, Vieira MM. A study on the relationship between mouth breathing and facial morphological pattern. Braz J Otorhinolaryngol. 2007;73(4):500-5.

9. Cattoni DM, Fernandes FD, Di Francesco RC, Latorre MRDO. Characteristics of the stomatognathic system of mouth breathing children: anthroposcopic approach. Pró Fono. 2007;19(4):347-51.

10. Marchesan, IQ. Avaliação e Terapia dos Problemas da Respiração. In: Marchesan, IQ. Fundamentos em Fonoaudiologia: Aspectos Clínicos da Motricidade Oral. Rio de Janeiro: Guanabara-Koogan; 1998. p. 23-36.

11. Branco A, Ferrari GF, Weber SA. Alterações orofaciais em doenças alérgicas de vias aéreas. Rev Paul Pediat. 2007;25(3):266-70.
12. Gouveia SAdS, Nahás FCR, Cotrim-Ferreira FA. Estudo cefalométrico das alterações dos terços médio e inferior da face em pacientes com diferentes padrões respiratórios e tipos faciais. $R$ Dental Press Ortodon Ortop Facial. 2009;14(4):92-100.

13. Coelho AR, Tanaka O, Ribeiro JS, Machado MA, Camargo ES. Transverse craniofacial dimensions in Angle Class II, Division 1 malocclusion according to breathing mode. Braz Oral Res. 2010;24(1):70-5.

14. Ricketts RM, Roth RH, Chaconas SJ, Schulhof RJ, Engel GA. Orthodontic diagnosis and planning their roles in preventive and rehabilitative dentristy. 1 ed. Denver: Rocky Mountain; 1982.

15. Laine $\mathrm{T}$, Alvesalo L, Lammi S. Palatal dimensions in 45,X-females. J Craniofac Genet Dev Biol. 1985;5(3):239-46.

16. Oliveira MOd, Vieira MM. Influência da respiraçäo bucal sobre a profundidade do palato. Pró-Fono. 1999;11(1):13-20.

17. Järvinen $\mathrm{S}$. Occurrence of different facial types and their relationship to the maxillary arch width in a Finnish child population. A cross-sectional study. Acta Odontol Pediatr. 1981;2(1):11-5.

18. Kanashiro LK, Vigorito JW. Estudo das formas e dimensões das arcadas dentárias superiores e inferiores em leucodermas, brasileiros, com maloclusão de classe II-Divisão $1^{\text {a }}$ e diferentes tipos faciais. Ortodontia. 2000;33(2):8-18.

19. Freitas Fd, Bastos E, Primo L, Freitas Vd. Evaluation of the palate dimensions of patients with perennial allergic rhinitis. Int $\mathrm{J}$ Paediatr Dent. 2001;11(5):365-71.

20. Ghasempour M, Mohammadzadeh I, Garakani S. Palatal arch diameters of patients with allergic rhinitis. Iran J Allergy Asthma Immunol. 2009;8(1):63-4.

21. Feres MFN, Enoki C, Sobreira CR, Matsumoto MAN. Dimensões do palato e características oclusais de crianças respiradoras nasais e bucais. Pesq Bras Odontoped Clin Integr. 2009;9(1):25-9.

22. Cattoni DM, Fernandes FD. Anthropometric orofacial measurements of children from São Paulo and from North America: comparative study. Pró Fono. 2009;21(1):25-9.

23. Mateus AR, Dolci JE, Costa HO, Sousa FC, di Biase N. Experimental study on the influence of facial muscle activity on the facial mesostructure bones in rabbits. Braz J Otorhinolaryngol. 2008;74(5):685-90. 
24. Berwig LC, Montenegro MM, Ritzel RA, Silva AMT, Corrêa ECR, Mezzomo CL. Influence of the respiratory mode and nonnutritive sucking habits in the palate dimensions. Braz $\mathrm{J}$ Oral Sci. 2011;10(1):42-9
25. Mezzomo CL, Machado PG, Pacheco AdB, Gonçalves BFdT, Hoffmann CF. As implicações da classe II de angle e da desproporção esquelética tipo classe II no aspecto miofuncional. Rev CEFAC. 2010; ahead of print:0.

http://dx.doi.org/10.1590/S1516-18462011005000134

RECEBIDO EM: 08/04/2011

ACEITO EM: 08/06/2011

Endereço para correspondência:

Luana Cristina Berwig

Rua Araújo Viana, no 173, apto 101,

Nossa Senhora de Fátima - Santa Maria - RS

CEP: 97015-040

E-mail: luanaberwig@gmail.com 